\title{
Uma experiência de psicoterapia de grupo dentro da Estratégia Saúde da Família
}

\author{
An experience of group psychotherapy in the Brazilian Family Health Program \\ Una experiencia de psicoterapia de grupo dentro \\ de la Estrategia Salud de la Familia
}

Roy Abrahamian ${ }^{1 *}$

Palavras-chave: Estratégia Saúde da Família Psicoterapia de Grupo Análise Transacional

Terapia Cognitiva Saúde Mental

Keywords: Family Health Program Psychotherapy, Group Transactional Analysis

Cognitive Therapy

Mental Health

\section{Resumo}

Este trabalho avaliou um Grupo de Saúde Mental desenvolvido na Estratégia Saúde da Família, baseado na análise transacional associada à terapia cognitivo-comportamental, descrevendo 0 impacto desta intervenção na melhoria da qualidade de vida da população atendida. Na Unidade Básica de Saúde Cruz das Almas, da Prefeitura de São Paulo, desde janeiro de 2007, realiza-se um grupo de Saúde Mental, com a frequência de uma vez por semana, com duração de uma hora. Neste grupo, os participantes discutem temas de seus interesses e aprendem técnicas de psicoterapia para lidarem com eventos do cotidiano e manejarem suas emoções e comportamentos de forma construtiva. 0 grupo é aberto aos interessados em participar das sessões de terapia, constituindo-se, em sua maioria, de pacientes acompanhados pelas equipes do Programa Saúde da Família e encaminhados ao Grupo de Saúde Mental. Ao ingressarem ao grupo, os pacientes preenchem o questionário sobre a saúde do(a) paciente, versão brasileira, conhecida pela sigla PHQ-9. Após frequentarem o grupo por, no mínimo, 12 sessões, os participantes preenchem um questionário de autoavaliação, informando sobre sua sensação de melhora em relação à sua saúde, melhora na autoestima e nos relacionamentos pessoais, e a visão mais positiva da vida e das outras pessoas. Dos participantes avaliados, 40 preencheram o questionário PHQ-9. Destes, nove $(22,5 \%)$ tiveram pontuação de zero a quatro (sem depressão); seis (15\%) tiveram pontuação de cinco a nove (depressão leve); dez (25\%) tiveram pontuação de 10 a 14 (depressão moderada); sete (17,5\%) tiveram pontuação de 15 a 19 (depressão moderada a grave); e oito (20\%) tiveram pontuação de 20 ou mais (depressão grave). Dos 65 pacientes que responderam ao questionário de autoavaliação após pelo menos 12 sessões de terapia, 51 (78\%) relataram visão mais positiva da vida e dos outros com a terapia; $44(67 \%)$ referiram melhora da autoestima; 52 (80\%), melhora nos relacionamentos e $53(81 \%)$, melhora em relação à saúde. A melhora relatada pelos pacientes nas diversas áreas de suas vidas aponta para a importância desse tipo de abordagem dentro da Estratégia Saúde da Família, e para o impacto positivo da Psicoterapia baseada na Análise Transacional associada a técnicas cognitivo-comportamentais no tratamento de pacientes com transtornos mentais na Atenção Primária à Saúde.

\section{Abstract}

This article evaluated an experiment carried out at the Family Health Program with the establishment of a Mental Health Group, based on transactional analysis associated with the cognitive-behavioral therapy, describing the impact of this intervention in improving the quality of life of the population served by the public system. In the Cruz das Almas Health Basic Unit, from São Paulo City Hall, a group of Mental Health has been happening since January, 2007, with the frequency of once a week, during one hour. In it, participants discuss topics of interest and learn psychotherapy techniques to deal with daily events and to manage their emotions and behaviors in a constructive way. The group is open to all those interested in participating in therapy sessions, being constituted, in most cases, by

${ }^{1}$ Associação Saúde da Família; União Nacional dos Analistas Transacionais - Brasil. abrahamian@uol.com.br

*Autor correspondente.

Fonte de financiamento: Associação Saúde da Família.

Conflito de interesses: declarou não haver.

Recebido em: 17/06/2011

Aprovado em: 10/01/2012 
patients followed by the Family Health Program teams and sent to the Mental Health Group. When patients join the group, they fill out a questionnaire known as Patient Health Questionnaire, Brazilian version, known by the acronym PHQ-9. After attending the group for at least 12 sessions, participants complete a self-assessment questionnaire, informing about feeling better on their health, improved self-esteem, and personal relationships, and about having a more positive view of life and of other people. Of the evaluated participants, 40 completed the questionnaire. From these, nine (22.5\%) had scores of zero to four (without depression); six (15\%) scored five to nine (mild depression); ten (25\%) had scores from 10 to 14 (moderate depression); seven (17.5\%) had a score of 15 to 19 (moderate to severe depression); and eight (20\%) had scores of 20 or more (severe depression). Of the 65 patients who completed the questionnaire for self-evaluation after at least 12 sessions of therapy, 51 (78\%) reported a more positive view of life and of other people; 44 (67\%) reported improved self-esteem; 52 (80\%) related improvement in relationships and $53(81 \%)$ improvement in relation to their health. The improvement reported by patients in different areas of their lives points to the importance of this approach within the Family Health Strategy and the positive impact of psychotherapy based on transactional analysis associated with cognitive-behavioral techniques in treating patients with mental disorders within the Health Primary Attention.

Palabras clave: Estrategia de Salud de la Familia Psicoterapia de Grupo Análisis Transaccional

Terapia Cognitiva Salud Mental

\section{Resumen}

Este artículo evaluó un Grupo de Salud Mental desarrollado en la Estrategia de Salud de la Familia, basado en el análisis transaccional asociada con la terapia cognitivo-conductual, que describe el impacto de esta intervención en la mejora de la calidad de vida de la población atendida. En la Unidad Básica de Salud Cruz das Almas, de la Municipalidad de São Paulo, ha estado funcionando desde enero de 2007 un grupo de Salud Mental, con la frecuencia de una vez por semana, con una duración de una hora. En este grupo, los participantes discuten temas de su interés y aprenden técnicas de psicoterapia para hacer frente a los acontecimientos cotidianos y gestionar sus emociones y conductas de una manera constructiva. El grupo está abierto a todos los interesados en participar en las sesiones de terapia, siendo, en su mayoría, pacientes seguidos por los equipos de la del Programa Salud de la Familia y enviados al Grupo de Salud Mental. Al participar en el grupo, los pacientes completan el cuestionario sobre la salud del paciente, versión brasileña, conocida como el PHQ-9. Después de asistir al grupo por lo menos 12 sesiones, los participantes llenan un cuestionario de auto-evaluación, informando sobre su sensación de mejora en relación con su salud, la mejora de la autoestima y las relaciones personales y sobre su visión más positiva de la vida y de las otras personas. De los participantes evaluados, 40 completaron el cuestionario PHQ-9. De estos, nove (22,5\%) tuvieron una puntuación de cero a cuatro (sin depresión); seis (15\%) tuvieron una puntuación de cinco a nove (depresión leve); dez (25\%) tuvieron una puntuación de 10 a 14 (depresión moderada); siete (17,5\%) tuvieron una puntuación de 15 a 19 (depresión moderada a severa); y ocho (20\%) tuvieron puntuaciones de 200 más (depresión severa). De los 65 pacientes que completaron el cuestionario de auto-evaluación después de al menos 12 sesiones de terapia, 51 (78\%) reportaron una visión más positiva de la vida y de las otras personas; 44 (67\%) reportaron mejora de la autoestima; 52 (80\%) reportaron mejora en las relaciones y 53 (81\%), mejora en relación con su salud. La mejora reportada por los pacientes en las diferentes áreas de sus vidas muestra la importancia de este enfoque dentro de la Estrategia de Salud de la Familia y para el impacto positivo de la Psicoterapia basada en el Análisis Transaccional asociada con técnicas cognitivo-conductuales en el tratamiento de pacientes con trastornos mentales en la Atención Primaria de Salud.

\section{Introdução}

Estudos realizados na área de saúde mental têm apontado que aproximadamente $30 \%$ das pessoas sofrem, em algum momento de suas vidas, um transtorno mental, como transtornos de ansiedade, depressão, alcoolismo, entre outros ${ }^{1}$. Além disso, sabe-se, atualmente, que, mesmo no caso de doenças de base orgânica, como diabetes, artrite reumatoide, asma, hipertensão arterial, doenças inflamatórias intestinais e câncer, os fatores emocionais desempenham forte influência no quadro clínico e no prognóstico dos pacientes. Segundo Mari e Jorge ${ }^{2}$, "os levantamentos epidemiológicos populacionais realizados no Brasil apontam para uma prevalência de transtornos mentais aproximada de $30 \%$, na população adulta, no período de um ano. Quando a prevalência de transtornos mentais é ajustada para os casos que demandam algum tipo de cuidado médico, chega-se a uma estimativa aproximada de $20 \%$, ou seja, um em cada cinco adultos demanda algum tipo de atenção em saúde mental num período de 12 meses. \{...\} Cerca de 2/3 dos estudos que avaliaram morbidade com instrumentos padronizados colocaram a prevalência de transtornos mentais na clínica geral entre 27 e $48 \%$ ".

Segundo Cordioli e Manfro ${ }^{3}$, entre os transtornos mentais, destacam-se os de ansiedade, ocorrendo em 3 a 17\% da população, e os depressivos, com prevalência variando entre 3 e $11 \%$, de acordo com Fleck ${ }^{4}$. Devido à alta frequência de portadores de transtornos mentais na população em geral, torna-se necessária, dentro da Estratégia Saúde da Família (ESF), uma abordagem sistêmica dos pacientes, que leve em consideração não apenas seus sintomas, mas também permita o compartilhamento de experiências e valorize os aspectos psicológicos e sociais dos pacientes e de suas famílias.

O objetivo deste trabalho foi descrever o impacto de um grupo de saúde mental desenvolvido na Unidade Básica de Saúde (UBS) Cruz das Almas, baseado na análise transacional associada à terapia cognitivo-comportamental, descrevendo o impacto desta intervenção na melhoria da qualidade de vida da população atendida. 


\section{Metodologia}

Trata-se de um estudo descritivo com informações coletadas de questionários preenchidos pelos pacientes integrantes do Grupo de Saúde Mental da UBS Cruz das Almas, entre janeiro de 2009 a agosto de 2011. Foram estudados 65 pacientes ao longo deste período, sendo que 40 preencheram o questionário PHQ-9 e todos preencheram o questionário de autoavaliação.

Na UBS Cruz das Almas, o grupo de saúde mental é realizado semanalmente, com uma hora de duração, desde janeiro de 2007. O grupo recebe os pacientes encaminhados pelos médicos generalistas da UBS, pelo Centro de Atenção Psicossocial (CAPS) e por outros serviços, como os de Pronto-Socorro de Psiquiatria, hospitais e ambulatórios psiquiátricos e gerais. O grupo é aberto, sendo, em sua maioria, constituído por pacientes portadores de transtornos de ansiedade, depressão, transtornos psicóticos compensados, dependência química e alcoolismo.

Ao ingressarem ao Grupo de Saúde Mental, os pacientes preenchem o Questionário sobre a Saúde do(a) Paciente-9, versão brasileira, conhecido pela sigla PHQ-9, como forma de triagem de depressão.

Após frequentarem o grupo por no mínimo 12 sessões, os participantes preenchem um questionário de autoavaliação, informando sobre a sensação de melhora em relação à saúde, melhora na autoestima e nos relacionamentos pessoais e uma visão mais positiva da vida e das outras pessoas.

Durante as sessões, são discutidos os principais tópicos da análise transacional e são abordadas técnicas cognitivocomportamentais aplicadas ao tratamento dos principais transtornos mentais, como os de ansiedade, depressão, transtornos psicóticos, alcoolismo e a dependência química.

A análise transacional é um método criado pelo psiquiatra canadense Eric Berne, em 1958. Consiste em uma teoria da Psicologia individual e social, baseada na análise dos estados de ego (componentes da personalidade humana), nos tipos de transações (comunicações entre as pessoas) e nos jogos psicológicos (comportamentos disfuncionais resultantes de relacionamentos não-saudáveis entre as pessoas). A análise transacional constitui também uma filosofia de vida que considera todo ser humano adequado, ou de valor, em sua essência e merecedor de ser tratado com amor e dignidade ${ }^{5,6}$.

A terapia cognitivo-comportamental baseia-se, fundamentalmente, no modelo cognitivo desenvolvido por Aaron Beck. De acordo com este modelo, as emoções e os comportamentos de uma pessoa são fortemente influenciados por suas crenças e pensamentos. Portanto, de acordo com Beck, ajudar o paciente a reformular suas crenças e pensamentos, tornando-os mais racionais e realistas, é fun- damental no tratamento dos transtornos mentais, como a ansiedade e a depressão ${ }^{7}$.

Os temas são abordados de forma interativa com os participantes, que expõem suas experiências de vida, compartilham suas visões de mundo, trocam opiniões a respeito de seus problemas e debatem métodos para resolução de problemas. Ao ingressarem no grupo, os pacientes são orientados quanto aos princípios da Psicoterapia em grupo e formulam um contrato de tratamento, de acordo com os postulados da análise transacional. De acordo com este ramo da Psicoterapia, o contrato consiste em uma meta objetiva de mudança positiva do comportamento, que seja verificável tanto pelo paciente como pelo grupo e pelo terapeuta. Os pacientes costumam expor seu contrato ao grupo e recebem "carícias" (sinais de atenção e reconhecimento), quando avançam na terapia e cumprem seu contrato.

De forma geral, os temas abordados nos grupos seguem os tópicos fundamentais da análise transacional e da terapia cognitivo-comportamental, a saber: aprendizado do modelo cognitivo, registro de pensamentos disfuncionais, reestruturação cognitiva, aprendizado de habilidades sociais, resolução de problemas, aprendizado dos estados de ego, manejo das emoções e comportamentos disfuncionais, técnicas de relaxamento e meditação.

Os grupos são ministrados por um médico generalista com formação em análise transacional e contam com a colaboração de agentes comunitários de saúde, com participação eventual de enfermeiros, auxiliares de Enfermagem e membros do Núcleo de Apoio à Saúde da Família (NASF).

O presente estudo foi aprovado por Comissão de Ética em Pesquisa da UBS Cruz das Almas - Associação Saúde da Família.

\section{Resultados}

Dos 65 participantes do Grupo de Saúde Mental que foram estudados, 40 preencheram o questionário PHQ-9, obtendo-se os resultados expostos na Tabela 1.

Dos 65 pacientes que preencheram o questionário de autoavaliação com pelo menos 12 sessões de terapia de grupo, obtiveram-se os resultados expostos na Tabela 2 .

Tabela 1. Resultados do preenchimento do PHQ-9.

\begin{tabular}{lcc}
\hline Grau de depressão & $\mathrm{n}(40)$ & $\%$ \\
\hline Sem depressão (0-4 pontos) & 9 & 22,5 \\
Depressão leve (5-9 pontos) & 6 & 15 \\
Depressão moderada (10-14 pontos) & 10 & 25 \\
Depressão moderada a grave (15-19 pontos) & 7 & 17,5 \\
Depressão grave (20 ou mais pontos) & 8 & 20 \\
\hline
\end{tabular}


Tabela 2. Resultados do questionário de autoavaliação.

\begin{tabular}{lcc}
\hline \multirow{2}{*}{ Perguntas } & \multicolumn{2}{c}{ Respostas positivas } \\
& $\mathrm{n}(65)$ & $\%$ \\
\hline $\begin{array}{l}\text { Depois que você começou a frequentar o grupo de saúde } \\
\text { mental, você sentiu melhora em relação à sua saúde? }\end{array}$ & 53 & 81 \\
( ) Sim ( ) Não & & \\
$\begin{array}{l}\text { Depois que você começou a frequentar o grupo de saúde mental, você } \\
\text { sentiu melhora na sua capacidade de se relacionar com as pessoas? }\end{array}$ & 52 & 80 \\
( ) Sim ( ) Não & & \\
$\begin{array}{l}\text { Depois que você começou a frequentar o grupo de saúde } \\
\text { mental, você melhorou sua autoestima? }\end{array}$ & 44 & 67 \\
( ) Sim ( ) Não \\
$\begin{array}{l}\text { Depois que você começou a frequentar o grupo de saúde } \\
\text { mental, você passou a enxergar os seus problemas e o mundo } \\
\text { de uma maneira mais positiva? }\end{array}$ \\
\begin{tabular}{l} 
( ) Sim ( ) Não \\
\hline
\end{tabular}
\end{tabular}

\section{Discussão}

No presente estudo, detectou-se, pelo questionário PHQ9, um índice considerável de pacientes com transtorno depressivo, os quais procuraram o Grupo de Saúde Mental $(77,5 \%)$, com destaque para as formas moderadas, moderadas a graves e graves $(62,5 \%)$. Dos 65 pacientes que responderam ao questionário de autoavaliação após no mínimo 12 sessões de Psicoterapia de grupo, 78\% relataram uma visão mais positiva da vida e dos outros, $67 \%$ referiram melhora na autoestima, $80 \%$ relataram melhora nos relacionamentos e $81 \%$, melhora na saúde. Com base no número limitado de pessoas analisadas ao longo do período estudado, os dados do presente trabalho sugerem que os pacientes são beneficiados pela realização de um Grupo de Saúde Mental, utilizando a Psicoterapia baseada na análise transacional com técnicas cognitivo- -comportamentais, tais como o registro de pensamentos disfuncionais, as técnicas de relaxamento, a monitoração do humor e a resolução de problemas.

\section{Conclusões}

A partir dos dados analisados no presente estudo, concluiu-se que a Psicoterapia de grupo, baseada na análise transacional, associada a técnicas cognitivo-comportamentais pode ser eficaz no manejo dos principais transtornos mentais, particularmente a ansiedade e a depressão, constituindo-se em um instrumento útil no tratamento de tais distúrbios dentro da ESF.

\section{Referências}

1. Andrade LHSG, Wang YP. Epidemiologia dos transtornos psiquiátricos. In: Alvarenga PG, Andrade AG. Fundamentos em Psiquiatria. Barueri: Manole; 2008. p. 68

2. Mari J de J, Jorge MR. Epidemiologia dos Transtornos Mentais. In: Mari J de J, Razzouk D, Peres MFT, Del Porto JA. Guias de Medicina Ambulatorial e Hospitalar da Escola Paulista de Medicina da Universidade Federal de São Paulo - Psiquiatria. Barueri: Manole; 2005. p. 1-2.

3. Cordioli AV, Manfro GG. Transtornos de Ansiedade. In: Duncan BB, Schmidt MI, Giugliani E. Medicina ambulatorial: condutas de Atenção Primária Baseadas em Evidências. 3. ed. Porto Alegre: Artmed; 2004. p. 864.

4. Fleck MP de A. Depressão. In: Duncan BB, Schmidt MI, Giugliani ERJ. Medicina ambulatorial: Condutas de Atenção Primária Baseadas em Evidências. 3. ed. Porto Alegre: Artmed; 2004. p. 874.

5. Berne E. Análise Transacional em Psicoterapia. 2. ed. São Paulo: Summus Editorial; 1985. 243 p.

6. Berne E. O que você diz depois de dizer Olá? São Paulo: Nobel; 1988 $357 \mathrm{p}$.

7. White JR, Freeman AS. Terapia cognitivo-comportamental em grupo para Populações e Problemas Específicos. São Paulo: Roca; 2003. 460 p. 